\title{
Lateral wall osteotomy combined with embedded biodegradable implants for displaced intra-articular calcaneal fractures
}

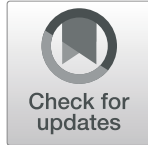

\author{
Yang Yang ${ }^{1 \dagger}$, Xiaoxiao Zhou ${ }^{2 \dagger}$, Mengqin Zhang ${ }^{3}$, Yichi Zhou ${ }^{4}$, Bin Wang ${ }^{1 *}$ and Chiting Yuan ${ }^{1}$
}

\begin{abstract}
Background: The extensile lateral approach (ELA) has been widely used to treat displaced intra-articular calcaneal fractures (DIACFs) and remains the gold standard procedure. Orthopedic surgeons are extremely concerned of the high rate of wound complications. This study intended to report a new surgical technique of the lateral wall osteotomy combined with an embedded biodegradable implant for treating DIACFs and assess clinical and radiological results.
\end{abstract}

Methods: From May 2013 to December 2015, a total of 17 patients with 19 calcaneal fractures underwent surgical treatment using our new technique. Radiographic images, computed tomography (CT) scans, and magnetic resonance (MR) images of the operative limb were obtained to assess fracture healing and biodegradable implant degradation. American Orthopaedic Foot and Ankle Society (AOFAS) ankle/hindfoot score at the last follow-up was obtained to assess functional result for all cases. Böhler's and Gissane's angles, width, and height of the injured calcaneus were analyzed using preoperative and last follow-up radiographic images.

Results: All radiological parameters were significantly improved th the last follow-up, with an increase of $15.58^{\circ}$, $8.38^{\circ}$, and $7.65 \mathrm{~mm}$ in Böhler's angle, Gissane's angle, and calcaneal height, respectively, and a decrease of $2.51 \mathrm{~mm}$ in calcaneal width $(p<0.05)$. Mean AOFAS score at the last follow-up was $84.37 \pm 9.98$, with 9,6 , and 4 feet, having excellent, good, and fair rates, respectively. None had nonunion, delayed union, or malunion after a mean follow-up of $34.69 \pm 5.22$ months. One superficial infection occurred 6 days post-surgery.

Conclusions: Osteotomy of the lateral wall of the calcaneus allows tension-free suturing and avoids damage to penetrating branches of the lateral calcaneal artery (LCA). Biodegradable implants are easy to reshape and do not require surgical removal. However, they should be limited to Sander's type II and III fractures only.

Level of evidence: Level IV, case series without controls

Keywords: Calcaneal fractures, Lateral wall osteotomy, Biodegradable implants, Wound complications, Outcomes

\section{Background}

Calcaneal fractures are among the most common tarsal fractures, approximately $75 \%$ of which are displaced intra-articular calcaneal fractures (DIACFs) [1]. The extensile lateral approach (ELA) has been widely used to treat DIACFs and remains the gold standard procedure for calcaneal fracture surgery owing to its excellent

\footnotetext{
* Correspondence: wangb1238@163.com

Yang Yang and Xiaoxiao Zhou are co-first authors.

'Department of Orthopedics, Taizhou Hospital of Zhejiang Province, Affiliated to Wenzhou Medical University, Linhai, Zhejiang, China

Full list of author information is available at the end of the article
}

visualization of the subtalar joint, sinus tarsi, and calcaneocuboid joint, and its easy implant placement [2]. Orthopedic surgeons are extremely concerned of the high rate of wound complications, including superficial, deep infection, skin flap necrosis, and wound dehiscence, which have been reported to be as high as $24 \%$ [3].

The impaired microvascularization of the posterior lateral skin flap that resulted from the initial or iatrogenic injury was considered the cause of numerous wound-healing complications, because the lateral calcaneal artery (LCA), derived from the posterior peroneal artery, is responsible for blood supply to the posterior lateral area of the foot. To

(C) The Author(s). 2019 Open Access This article is distributed under the terms of the Creative Commons Attribution 4.0 International License (http://creativecommons.org/licenses/by/4.0/), which permits unrestricted use, distribution, and 
avoid these vexing complications, the microvascularization of the posterior lateral skin flap should be protected. The minimal invasion approach or percutaneous reduction could avoid damaging LCA. However, achieving the ideal reduction and stable fixation of calcaneal fractured fragments is difficult in these procedures, thereby leading to poor clinical outcomes $[4,5]$. Moreover, some modifications of ELA were proposed, such as making a curved corner of the incision to decrease the wound complications rates, and several new wound closure techniques were also devised, but failed to avoid injury of LCA [6]. Meanwhile, different metallic implants were designed for minimally invasive approaches or other newly developed approaches. However, secondary surgical procedures for metallic implant removal were also a wound-healing risk. Fixation strength of biodegradable implants was described previously, and its safety and effects at different terms were also confirmed [7-9].

To reduce wound complications after ELA, we devised a new technique of lateral wall osteotomy combined with embedded biodegradable plate and screws for treating DIACFs. This study aimed to present this new surgical technique and report our preliminary results.

\section{Materials and methods \\ Patients}

From May 2013 to May 2015, a total of 17 patients with 19 calcaneal fractures underwent surgical treatment with our new surgical technique after obtaining permission from the institutional ethical committee. The inclusion criteria were as follows: fresh closed calcaneal fractures, intro-articular calcaneal fractures confirmed by per-operative three-dimensional CT reconstruction, gap between fractures $>3 \mathrm{~mm}$ and/or step-off $>2 \mathrm{~mm}$, the width or height of the calcaneus changed significantly, decreased Böhler's and Gissane's angles, and patients who agreed to undergo biodegradable plate implantation and who signed the informed consent. Patients aged < 18 years, with open fracture, diabetes mellitus, lower extremity thromboangiitis obliterans, serious osteoporosis, or other serious low limb fractures were excluded. Preoperative and last follow-up radiographic images of the lateral and axial positions and three-dimensional reconstruction CT scans and MR images at the last follow-up were obtained to measure the radiological parameters. The timing of surgery depends on the presence of the wrinkle sign to assess whether skin wrinkling was present and pitting edema was absent. The injured limbs were inspected routinely to check for the wrinkle sign.

\section{Surgical technique \\ Skin incision}

Patients were placed in the contralateral decubitus position of the injured limb on a radiolucent operating table under general anesthesia or epidural anesthesia. Following the exsanguination of the injured limb, a hemostatic tourniquet was used. A transverse L-shaped extended lateral incision was made to expose the subtalar joints and fractured fragments. Vertical incision was done at the posterior one-third distance between the posterior aspect of the fibula and the anterior margin of the Achilles tendon. Then, a curved angle, about $90-100^{\circ}$, was used to avoid potential flap necrosis as a sharp angle would increase ischemia risk of the lateral flap edge at the corner, resulting in flap necrosis. The horizontal incision was placed at the junction of the hyperkeratotic skin of the lateral aspect transformed to the glabrous skin of the plantar aspect of the foot, aimed just on top of the fifth metatarsal and then curved to the base of the fourth metatarsal (Fig. 1). Care is taken to avoid injury to the sural nerve when cutting the skin at the proximal vertical and distal horizontal limbs of the incision because of the course of the sural nerve.

\section{Osteotomy and fixation}

A 1-2-mm-thick osteotomy was made in the lateral wall of the calcaneus with a sharp and wide osteotome. The bone flap that contained the integrated skin and subcutaneous tissue was retracted to expose subtalar joints and the fractured fragments. The bone flap should contain the integrated skin and subcutaneous tissue. After the desired fracture reduction was achieved, a well-molded biodegradable plate was implanted and Kirschner wires were used to fixate the fractured fragments provisionally. C-arm X-ray images of the lateral, axial, oblique, and Brodén's positions of the calcaneus were obtained to determine if the anatomic geometry of calcaneus was restored. Then, the $3.1-\mathrm{mm}$ flat and low-profile head screws were driven into the prefabricated hole (Fig. 1). The 1.2-mm thick free-form biodegradable plate (Inion, Finland) was bathed in a $70^{\circ} \mathrm{C}$ thermostat filled with normal saline for 1 min to make the plate malleable. This malleable state continued for 10-15 s. The free-form plate was cut at the midline along its longitudinal axis, and the cutout was opened to make a Y-shaped plate after bathing (Fig. 2). The plate was embedded in the calcaneus under the bone flap (Fig. 1), and hot normal saline was used to help make the plate more suitable. The periosteal margin of the lateral wall was sutured using Vicryl 2-0 (Ethicon, Division of Johnson \& Johnson, Somerville, NJ) (Fig. 1). No wound drainage was required in all patients (Fig. 2).

\section{Postoperative management}

At $24 \mathrm{~h}$ post-surgery, wound healing was routinely monitored. Below knee plaster cast was applied for 4-6 weeks postoperatively. At 4-6 weeks postoperatively, patients were encouraged to perform non-weight-bearing 


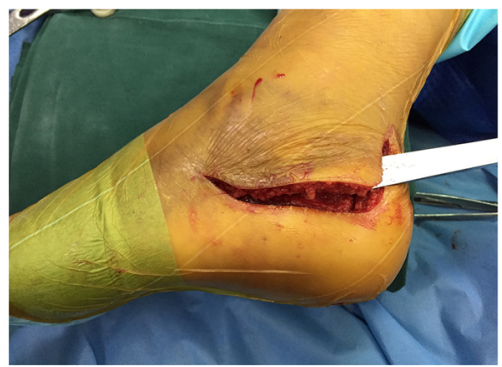

a

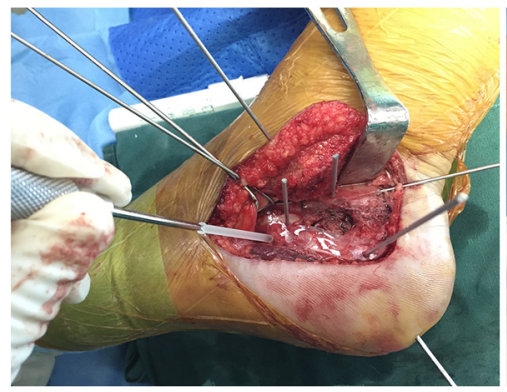

c

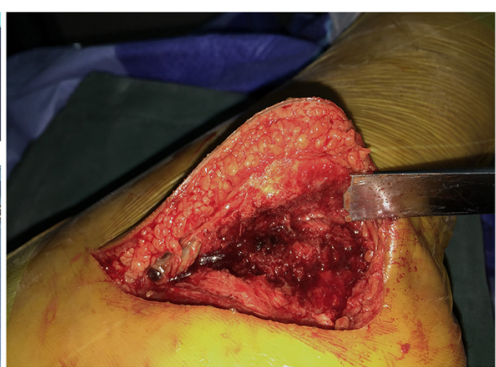

b

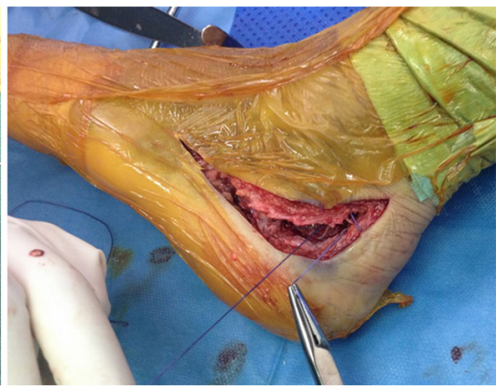

d

Fig. 1 Illustrations of the surgical procedures and technique. A transverse L-shaped extensile lateral incision and osteotomy of the lateral wall of the calcaneus with a sharp and wide osteotome are made. The bone flap containing the integrated skin and subcutaneous tissue is retracted, and the subtalar joint is exposed $(\mathbf{a}, \mathbf{b})$. After fracture reduction, the biodegradable plate is implanted and Kirschner wires are used to fixate the fractured fragments provisionally; then, the biodegradable screws are driven into the prefabricated holes (c). The periosteum is sutured with Vicryl 2-0 (d)

movements of the malleolus joints. After 8-10 weeks, partial weight-bearing movements with crutches were allowed. Patients were permitted to walk without crutches when bony union of the calcaneal fractured fragments was evident on the X-ray image.

\section{Methods of assessment}

The radiographic images, CT scans, and MR images of the operative limb were obtained to assess fracture healing and biodegradable implant degradation. Biodegradable implant absorption could be confirmed by the disappearance of screw holes on the radiographic images [10]. American Orthopaedic Foot and Ankle Society (AOFAS) ankle/hindfoot score at the last follow-up was employed to assess functional result for all cases. Radiological results were analyzed using preoperative and last follow-up radiographic images.

\section{Statistical analysis}

All data were analyzed using the SPSS version 21.0 (IBM Corp., Armonk, NY, USA). Continuous variables were presented as mean \pm standard deviation and were analyzed using paired $t$ test. Level of significance was set at 0.05 for all analyses.

\section{Results}

All radiological parameters were significantly improved at the last follow-up, with an increase of $15.58^{\circ}, 8.38^{\circ}$,

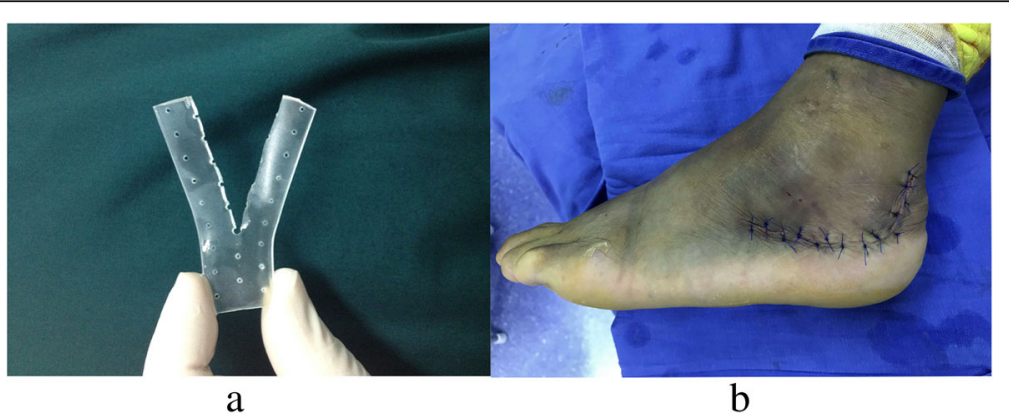

Fig. 2 The "Y" shape of the biodegradable plate (a), and the incision is closed using interrupted vertical mattress sutures without drains (b) 
and $7.65 \mathrm{~mm}$ in Böhler's angle, Gissane's angle, and calcaneal height, respectively, and a decrease of $2.51 \mathrm{~mm}$ in calcaneal width $(p<0.05)$ (Table 1$)$.

The mean AOFAS score at the last follow-up was $84.37 \pm 9.98$ (range, 65-95), with 9, 6, and 4 feet having excellent, good, and fair rates, respectively (Table 2). The excellent and good rates account for $78.95 \%$ of all cases. The 4 feet with fair AOFAS score were those of patients with Sander's type IV fracture. No nonunion, delayed union, or malunion was observed after a mean follow-up period of $34.69 \pm 5.22$ (range, 28-48) months. All returned to pre-injury work, and none required secondary surgery for implant removal.

\section{Complications}

Only one superficial infection occurred 6 days post-surgery, and it was resolved by cutting several intense sutures immediately, extending the administration of $1.5 \mathrm{~g}$ cefuroxime sodium twice a day for 5 days and changing dressings more frequently. One screw breakage was found in a patient at the last follow-up, which may be due to walking with weight-bearing earlier than we proposed. No gap $>3 \mathrm{~mm}$ or step-off $>2 \mathrm{~mm}$ of the subtalar joint and no paresthesia were found in all limbs. No soft tissue reaction was found in all feet at the final follow-up.

\section{Discussion}

Our findings revealed that our novel technique had a low rate of wound-healing complications. To avoid damage to the LCA, a vertical incision was done at the posterior one-third distance between the posterior border of the lateral malleolus and the lateral border of the Achilles tendon. Moreover, the full-thickness lateral flap, containing the lateral wall of calcaneus instead of peeling the lateral skin flap from the lateral wall, circumvented the disturbance of the sural nerve and the angiosome to the lateral skin flap. The LCA with a 6-mm diameter consists of an intricate vessel net surrounding the lateral hind foot and lateral malleolus and provides approximately $14 \%$ of blood supply to the skin flap in this area [11]. Several branches of the LCA penetrate the lateral wall of the calcaneus, accounting for approximately $45 \%$ of its blood supply [12]. Hence, the disturbance of the LCA caused by either initial or iatrogenic injury will diminish a large
Table 2 General data of all patients

\begin{tabular}{|c|c|c|}
\hline Item & Values & Range \\
\hline Sex (male/female) & $14 / 3$ & \\
\hline Age (year) & $46.72 \pm 8.56$ & $31-60$ \\
\hline Delayed time (day) & $5.79 \pm 2.23$ & $2-9$ \\
\hline Hospital stay duration (day) & $12.21 \pm 2.96$ & $6-20$ \\
\hline \multicolumn{3}{|l|}{ Sander's classification } \\
\hline$\|$ & 5 & \\
\hline III & 10 & \\
\hline IV & 4 & \\
\hline Follow-up (month) & $34.69 \pm 5.22$ & $28-48$ \\
\hline Operative limb (left/right/both) & $8 / 7 / 2$ & \\
\hline Bone graft $(n)$ & 7 & \\
\hline Wound complications ( $n$ ) & 1 & \\
\hline Screw breakage $(n)$ & 2 & \\
\hline Sural nerve lesion ( $n$ ) & 0 & \\
\hline AOFAS scores & $84.37 \pm 9.98$ & $65-95$ \\
\hline Excellent $(n)$ & 9 & \\
\hline Good $(n)$ & 6 & \\
\hline Fair $(n)$ & 4 & \\
\hline
\end{tabular}

Abbreviation: AOFAS American Orthopaedic Foot and Ankle Society

portion of blood supply to the lateral skin flap and calcaneus, increasing the likelihood of wound-healing complications and nonunion of the fractured calcaneus. As commonly described, the vertical limb of the incision was situated midway between the posterior edge lateral malleolus and lateral edge of the Achilles tendon, which would inevitably damage the LCA because the vertical incision was located around the course of the lateral calcaneal artery, and the tourniquet was used during the whole procedure [13, 14].

To avoid iatrogenic injury to the LCA, Elsaidy et al. introduced a dangerous triangle, which contained the superficial course of the LCA as the posterior border, and highlighted that the classically described vertical incision would cross this dangerous triangle and disturb the LCA [15]. Kwon et al. also found that a more posterior vertical incision decreased in fourfolds the risk of damaging the LCA compared to the classical ELA [16]. Theoretically, the osteotomy of the calcaneal lateral wall, instead of detaching the lateral soft tissue envelope from

Table 1 Preoperative and last follow-up radiological results

\begin{tabular}{lllll}
\hline Item & Preoperative & Last follow-up & Improvement & $p$ value \\
\hline Böhler's angle (degrees) & $9.82 \pm 8.47$ & $25.40 \pm 6.62$ & 15.58 & $<0.001$ \\
Gissane's angle (degrees) & $111.68 \pm 14.44$ & $120.06 \pm 13.90$ & 8.38 & 0.038 \\
Height (mm) & $41.47 \pm 4.37$ & $49.12 \pm 4.14$ & 7.65 & $<0.001$ \\
Width (mm) & $44.78 \pm 3.73$ & $42.27 \pm 2.70$ & 2.51 & 0.005 \\
\hline
\end{tabular}

All values are expressed as mean \pm standard deviation. Differences are considered significant at $p<0.05$ 


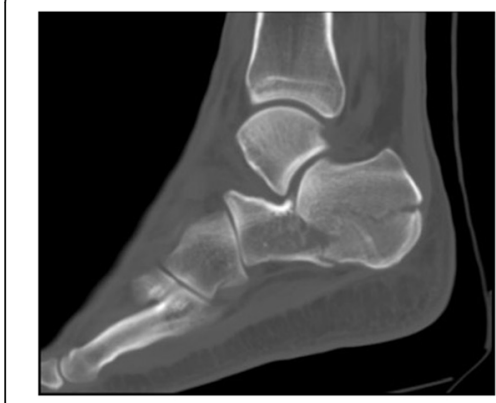

a

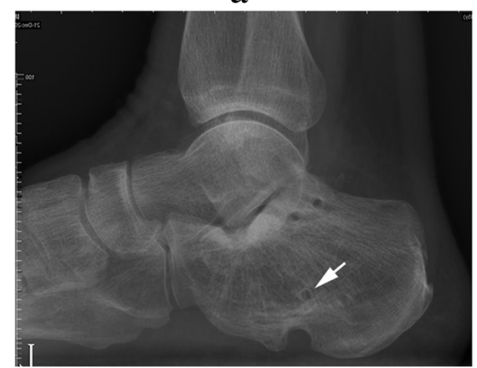

c

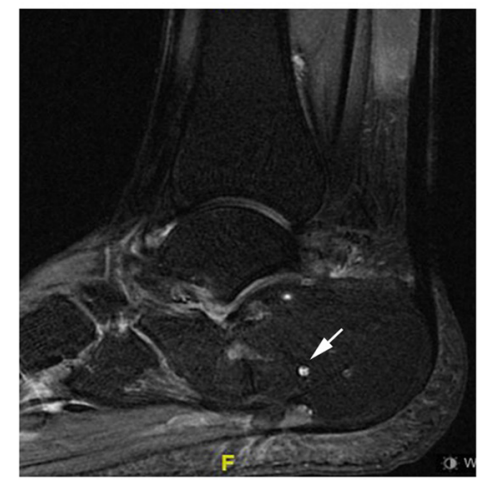

e

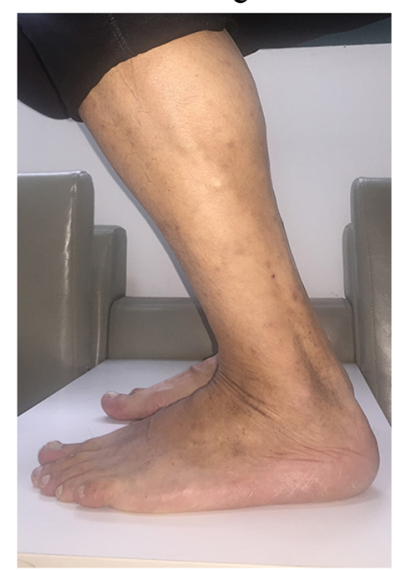

g

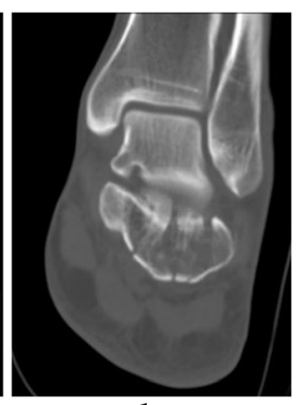

b

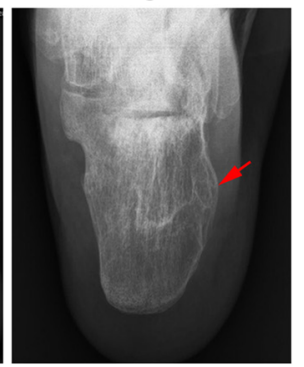

d

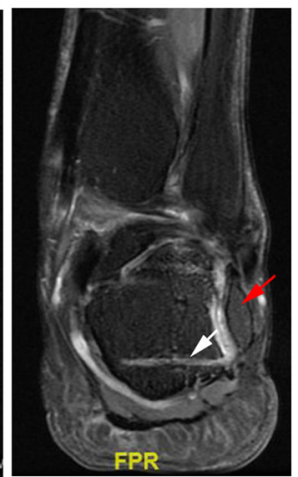

f

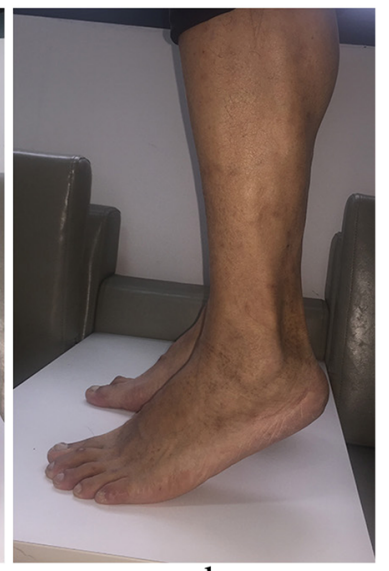

$\mathrm{h}$
Fig. 3 Radiographs of a 43-year-old male patient admitted for left calcaneal fractures of Sander's type III (a, b). Lateral (c) and axial (d) views at 36 months post-surgery showing bony union of the fracture and the lateral wall (red arrow); several screw holes remain (white arrow). Sagittal and coronal MR images (e, $\mathbf{f}$ ) showing partial absorption of biodegradable implants. The extension range of motion $(\mathbf{g})$ and the flexion range of motion (h) at the final follow-up are displayed

the lateral wall of calcaneus, allows tension-free retraction during the operation with the support of lateral wall to avoid injury to the penetrating branches of the lateral calcaneal artery, eliminate edema between the lateral wall of calcaneus and lateral skin flap, and remove the dead space between the lateral skin flap and implants.

In our previous study, the metallic plate and screws were applied to obtain rigid fixation of the fractured calcaneus [17]. Inherent demerits of embedding the metal implants in the osteotomy surface are as follows: removing implants is difficult with this procedure and there would be a residual dead space between the lateral wall and the implants. In an attempt to address these problems, we used biodegradable implants to fix calcaneal fractures. To the best of our knowledge, this is the first report on treating DIACFs with biodegradable implants. Biodegradable implants have been used for years in the treatment of less stressful fractures, such as ankle, mandibular, and pediatric clavicular fractures, with excellent results [18-20]. The less fixation strength of the biodegradable implant utilized for the fractures of the calcaneus may lead to stability problems of fracture fixation. Thus, weight-bearing activities were not allowed until there was bony union on radiographs. However, in our study, screw breakage occurred in one patient because of early weight-bearing exercises. Biodegradable implants maintain its stiffness for about 18 weeks, which is long enough for fracture union [7]. After 18 weeks, the stress shifts to the calcaneus gradually as the implant degrades slowly, resulting in less stress-shielding as compared to metallic implants and enabling dense bone formation [21]. The clinical result showed that the excellent and good rates of AOFAS score account for $78.95 \%$ of all cases (mean score, $84.37 \pm 9.98$; range, 65-95). In the present series, four Sander's type IV fractures were included, which may be associated with poor clinical results. No more screw breakage was observed in this case series at the final follow-up. Therefore, we believed that the fixation of biodegradable implants was strong enough, as we were able to prolong the period of weight-bearing-free joint exercises (Fig. 3). According to the findings of this study, the indications of this approach were Sander's type II and III closed calcaneal fractures. However, this approach is contraindicated in patients with Sander's type IV fractures, open fractures, 
severe osteoporosis, diabetes mellitus, and serious injuries.

One superficial infection was observed (5.26\%), which was lower than that previously reported [22]. This infected foot in the present study was classified as Sander's IV type and had no preoperative Doppler examination [23]. Thus, we could not determine whether the superficial infection was caused by preoperative injury to the LCA or iatrogenic damage or was a highly comminuted fracture itself. No calcaneal fracture nonunion or malunion was found in the present study.

The advantages of our developed technique include primary wound-healing tendency, complete visualization of fractured fragments, posterolateral subtalar joint facet and calcaneocuboid joint facet, no requirement for secondary operation for implant removal, and less stress-shielding of the calcaneus, which is beneficial for bony union. Its main disadvantage is that it has less fixation strength of biodegradable implants leading to delayed weight-bearing rehabilitation, which may result in poor clinical outcomes.

Major limitations of the present study include its relatively small patient sample size, and inclusion of two patients with bilateral fractures that made the comparison of both sides impossible, and patients with Sander's type IV and bilateral calcaneal fractures that may be caused by severe trauma, which may result in poor radiological and clinical outcomes. Furthermore, given that a comparison group treated using classical ELA with metallic implants was absent, we cannot confirm if this new approach is superior to classical ELA for all types of fractures. Moreover, as the fixation strength of biodegradable implants is weaker than metallic implants, according to findings of present study and our experience, our new surgical technique is not fit for Sander's type IV fractures. A proper prospective randomized controlled clinical study with long-term follow-up and a large sample size is needed to determine the safety and reliability of this surgical technique.

\section{Conclusions}

Osteotomy of the lateral wall of calcaneus allows tension-free suturing and avoids damage to penetrating branches of the LCA. Biodegradable implants are easy to reshape and do not require surgical removal. However, they should be limited to Sander's type II and III fractures only.

\section{Abbreviations \\ AOFAS: American Orthopaedic Foot and Ankle Society; CT: Computed tomography; DIACFs: Displaced intra-articular calcaneal fractures; ELA: Extensile lateral approach; LCA: Lateral calcaneal artery; MR: Magnetic resonance}

\section{Acknowledgements}

We would like to thank Editage [www.editage.cn] for the English language editing.

\section{Funding}

This work was funded by Projects of Medical and Health Technology Program of Zhejiang Province (2018PY079), Projects of Medical and Health Technology Program of Zhejiang Province (2015KYB443), Projects of Medical and Health Technology Program of Zhejiang Province (2014KYA226), and Natural Science Foundation of Shanghai (16ZR1431600). Fund for the Most Important Discipline Construction of Zhoupu Hospital of Shanghai Pudong New District (ZP-XK-2015a-2).

\section{Availability of data and materials}

The datasets used and/or analyzed in the present study are included in this published article.

\section{Authors' contributions}

BW and XXZ contributed to the experiment conception and design. MQZ contributed to the radiographic measurements and assessments. YY and CTY contributed to the postoperative clinical assessments and data analysis. YY and YCZ contributed to the writing of the manuscript. All authors have read and approved the final manuscript.

\section{Ethics approval and consent to participate}

This study was approved by the institutional review board of Taizhou Hospital of Zhejiang Province. All patients gave written informed consent before participating in this study.

\section{Consent for publication}

All patients gave their written informed consent for the publication of their individual data and identifying photographs.

\section{Competing interests}

The authors declare that they have no competing interests.

\section{Publisher's Note}

Springer Nature remains neutral with regard to jurisdictional claims in published maps and institutional affiliations.

\section{Author details}

'Department of Orthopedics, Taizhou Hospital of Zhejiang Province, Affiliated to Wenzhou Medical University, Linhai, Zhejiang, China. ${ }^{2}$ Department of Orthopedics, Zhoupu Hospital, Affiliated to Shanghai University of Medicine \& Health Sciences, Shanghai, China. Intensive Care Unit, Taizhou Hospital of Zhejiang Province, Affiliated to Wenzhou Medical University, Linhai, Zhejiang, China. ${ }^{4}$ Department of Orthopedics, CR \& WISCO General Hospital, Wuhan, Hubei, China.

Received: 8 November 2018 Accepted: 21 February 2019

Published online: 06 March 2019

\section{References}

1. Epstein N, Chandran S, Chou L. Current concepts review: intra-articular fractures of the calcaneus. Foot Ankle Int. 2012;33:79-86.

2. Sanders R, Fortin P, DiPasquale T, Walling A. Operative treatment in 120 displaced intraarticular calcaneal fractures. Results using a prognostic computed tomography scan classification. Clin Orthop Relat Res. 1993;290: 87-95.

3. Koski A, Kuokkanen H, Tukiainen E. Postoperative wound complications after internal fixation of closed calcaneal fractures: a retrospective analysis of 126 consecutive patients with 148 fractures. Scand J Surg. 2005;94:243-5.

4. Bai L, Hou YL, Lin GH, Zhang X, Liu GQ, Yu B. Sinus tarsi approach (STA) versus extensile lateral approach (ELA) for treatment of closed displaced intra-articular calcaneal fractures (DIACF): a meta-analysis. Orthop Traumatol Surg Res. 2018;104:239-44.

5. Park J, Che JH. The sinus tarsi approach in displaced intra-articular calcaneal fractures. Arch Orthop Trauma Surg. 2017;137:1055-65.

6. Hollawell S. Wound closure technique for lateral extensile approach to intraarticular calcaneal fractures. J Am Podiatr Med Assoc. 2008;98:422-5.

7. Väänänen $P$, Nurmi JT, Lappalainen $R$, Jank $S$. Fixation properties of a biodegradable "free-form" osteosynthesis plate with screws with cut-off screw heads: biomechanical evaluation over 26 weeks. Oral Surg Oral Med Oral Pathol Oral Radiol Endod. 2009;107:462-8. 
8. Isyar M, Yilmaz I, Nusran G, Guler O, Yalcin S, Mahirogullari M. Safety of bioabsorbable implants in vitro. BMC Surg. 2015;15:127.

9. Amini AR, Wallace JS, Nukavarapu SP. Short-term and long-term effects of orthopedic biodegradable implants. J Long-Term Eff Med Implants. 2011;21: 93-122.

10. Kim NK, Nam W, Kim HJ. Comparison of miniplates and biodegradable plates in reconstruction of the mandible with a fibular free flap. Br J Oral Maxillofac Surg. 2015;53:223-9.

11. Geddes C, Tang M, Yang D, Morris S. Anatomy of the integument of the lower extremity. In: Blondeel PNMS, Hallock GG, Neligen PC, editors. Perforator flaps. St. Louis: Quality Medical Publishing; 2013. p. 694-7.

12. Andermahr J, Helling HJ, Landwehr P, Fischbach R, Koebke J, Rehm KE. The lateral calcaneal artery. Surg Radiol Anat. 1998;20:419-23.

13. Benirschke SK, Sangeorzan BJ. Extensive intraarticular fractures of the foot. Surgical management of calcaneal fractures. Clin Orthop Relat Res. 1993;292: $128-34$.

14. Borrelli J Jr, Lashgari C. Vascularity of the lateral calcaneal flap: a cadaveric injection study. J Orthop Trauma. 1999;13:73-7.

15. Elsaidy MA, El-Shafey K. The lateral calcaneal artery: anatomic basis for planning safe surgical approaches. Clin Anat. 2009;22:834-9.

16. Kwon JY, Gonzalez T, Riedel MD, Nazarian A, Ghorbanhoseini M. Proximity of the lateral calcaneal artery with a modified extensile lateral approach compared to standard extensile approach. Foot Ankle Int. 2017;38:318-23.

17. Wang $B$, Sun $L$, Dong $R$, et al. Inlayed plate via lateral bone flap approach for displaced intra-articular calcaneal fractures (in Chinese). Chin J Onhop Trauma. 2014:16:1033-7.

18. Osborn EJ, Farnsworth CL, Doan JD, Edmonds EW. Bioabsorbable plating in the treatment of pediatric clavicle fractures: a biomechanical and clinical analysis. Clin Biomech (Bristol, Avon). 2018;55:94-9.

19. Gaiarsa GP, Dos Reis PR, Mattar R Jr, Silva Jdos S, Fernandez TD. Comparative study between osteosynthesis in conventional and bioabsorbable implants in ankle fractures. Acta Ortop Bras. 2015;23:263-7.

20. An J, Jia P, Zhang Y, Gong X, Han X, He Y. Application of biodegradable plates for treating pediatric mandibular fractures. J Craniomaxillofac Surg. 2015:43:515-20.

21. Losken HW, van Aalst JA, Mooney MP, et al. Biodegradation of inion fastabsorbing biodegradable plates and screws. J Craniofac Surg. 2008:19:748-56.

22. Schepers T, Den Hartog D, Vogels LM, Van Lieshout EM. Extended lateral approach for intra-articular calcaneal fractures: an inverse relationship between surgeon experience and wound complications. J Foot Ankle Surg. 2013;52:167-71.

23. Bibbo C, Ehrlich DA, Nguyen HM, Levin LS, Kovach SJ. Low wound complication rates for the lateral extensile approach for calcaneal ORIF when the lateral calcaneal artery is patent. Foot Ankle Int. 2014:35:650-6.

Ready to submit your research? Choose BMC and benefit from:

- fast, convenient online submission

- thorough peer review by experienced researchers in your field

- rapid publication on acceptance

- support for research data, including large and complex data types

- gold Open Access which fosters wider collaboration and increased citations

- maximum visibility for your research: over $100 \mathrm{M}$ website views per year

At $\mathrm{BMC}$, research is always in progress.

Learn more biomedcentral.com/submissions 